\title{
CDK4/6 inhibitors for hormone receptor-positive HER2-negative metastatic breast cancer: does one size fit all?
}

\author{
Palma Fedele ${ }^{*, 1}$ \& Saverio Cinieri ${ }^{1}$ \\ ${ }^{1}$ Medical Oncology \& Breast Unit, 'A Perrino' Hospital, Brindisi, Italy \\ *Author for correspondence: Tel.: +39 083153 7217; Fax: +39 083 153 7918; minafedele@hotmail.com
}

“Among all the targeted agents explored, CDK4/6 inhibitors can be considered the most promising and currently CDK4/ 6 inhibitors are available for the treatment of hormone receptor positive metastatic breast cancer patients. Nevertheless, there are issues to overcome in order to optimize the targeted use of these agents."

First draft submitted: 8 August 2018; Accepted for publication: 4 April 2019; Published online: 10 June 2019

Keywords: CDK4/6 inhibitors $\bullet$ endocrine resistance $\bullet$ endocrine therapy $\bullet$ metastatic breast cancer

Estrogen receptors (ER) occur in 60-70\% of breast cancers, and endocrine therapy represents one of the most important systemic therapy for hormone receptor-positive breast cancer. However, after an initial response to hormonal therapy, most tumors develop resistance leading to disease progression [1]. Different mechanisms of endocrine resistance have been successfully identified involving downregulation of ER expression, ER mutations or altered expression of ER coregulators, expression of hormone-insensitive cellular clones during treatment, increased estrogen synthesis and activation of ER through other signaling pathways involved in cell survival and proliferation [2]. Moreover, two different types of endocrine resistances have been identified, almost exclusively based on a time criterion of the response to previous hormone therapy performed in the adjuvant or metastatic setting of the disease. 'Primary' or 'de novo' endocrine resistance is characterized by the relapse of the tumor within 12-24 months of adjuvant endocrine therapy, or by the progression to metastatic breast cancer (MBC) within 6 months. However, in 'secondary' or 'acquired' endocrine resistance, relapse may occur within 12 months of completing adjuvant endocrine therapy or after the first 6 months of endocrine therapy for MBC.

Preclinical data have shown that endocrine resistance is in most cases based on the crosstalk between the ER and intracellular signaling pathways. Therefore, manipulation of growth factor signaling networks has emerged as an attractive strategy to delay the onset, or potentially even overcome, endocrine resistance.

In recent years a number of clinical trials have explored the use of targeted agents to overcome endocrine resistance especially in MBC, these include HER2/neu antagonists, IGF1R inhibitors, tyrosine kinase inhibitors, multikinase inhibitors, histone deacetylase inhibitors, mTOR antagonists, PI3K inhibitors and CDK4/6 inhibitors [2].

Among all the targeted agents explored, CDK4/6 inhibitors can be considered the most promising and currently CDK4/6 inhibitors are available for the treatment of hormone receptor positive metastatic breast cancer patients [3]. Nevertheless, there are issues to overcome in order to optimize the targeted use of these agents.

CDKs regulate cell proliferation and coordinate the cell-cycle checkpoint response to DNA damage. CDK4/6 regulates the passage of cells through the cell cycle by controlling the retinoblastoma protein. Deviations in the CDKs - retinoblastoma protein pathway are common in breast cancer. Amplification of cyclin D1 has been found in $15-20 \%$ of human breast cancers, whereas overexpression of the protein has been found at higher percentages (up to $50 \%$ ) [2].

In the hypophosphorylated state, the $\mathrm{Rb}$ protein acts as an onco-suppressor and contributes to the regulation of the cell cycle at the G1-S checkpoint, preventing the transcription of genes that are required for passage in the $S$ phase - meaning the cell cycle is blocked in G1. In response to mitogenic signals, CDK4/6 forms a complex with 
its regulatory subunit, such as cyclin D1, which phosphorylates the Rb protein, reducing its activity of suppressing gene transcription. CDK4/6 inhibitors restore Rb activity and reinstate the brakes on cell-cycle progression [4].

Selective CDK4/6 inhibitors, including palbociclib, ribociclib and abemaciclib, have emerged as attractive antineoplastic agents because of the importance of CDK4/6 activity in regulating cell proliferation. Moreover, preclinical studies identified luminal ER-positive breast cancer subtype as being associated with sensitivity to CDK4/6 inhibitors.

Based on this preclinical data, the activity of CDK4/6 inhibitors was investigated in the clinical setting for the treatment of endocrine-sensitive and -resistant breast cancer.

Three Phase III studies, Paloma-2 [5], Monaleesa-2 [6] and Monarch-3 [7], have explored activity and safety of the three CDK4/6 inhibitors, palbociclib, ribociclib and abemaciclib, respectively. CDK4/6 inhibitors significantly increased median progression-free survival (PFS), which was the primary end point in all the three studies, versus endocrine therapy, by 10 months. Similarly CDK4/6 inhibitors significantly increased median PFS by approximately 7 months versus endocrine therapy alone when administered to endocrine-resistant breast cancer as second-line therapy [8-10].

Despite the efficacy in the first- and second-line treatments, many questions about optimal use of CDK4/6 inhibitors still remain unanswered.

We know that palbociclib, ribociclib and abemaciclib do not have equal mechanisms of action and differ in target, dosing regimen and in the side-effect profile.

Palbociclib has equivalent CDK4/cyclin D3 and CDK6/cyclin D1 potency, while both ribociclib and abemaciclib are significantly more potent toward CDK4/cyclin D3 (ribociclib is fivefold more potent; abemaciclib is ninefold more potent) [11].

Abemaciclib has monotherapy activity, can cross the blood-brain barrier and has some clinical activity on brain metastases.

Dosing regimen is intermittent, 3 weeks on and 1 week off for palbociclib and ribociclib - whereas abemaciclib is administered once a day, continuously.

Moreover, neutropenia is the most common side effect observed with palbociclib and ribociclib, while diarrhea is the most common adverse event observed with abemaciclib [12].

Since all the three selective CDK inhibitors were active in the treatment of ER-positive breast cancer, it is difficult to identify which is the first in the class in the absence of direct comparison studies among the three CDK4/6 inhibitors.

Furthermore, although CDK4/6 inhibitors have shown to overcome endocrine resistance mainly in breast cancer patients pretreated with endocrine therapy for early or metastatic disease, and with a disease-free interval (DFI) $>12$ months, we have very little data on the efficacy of these drugs in de novo endocrine-resistant breast cancer (such as those patients with DFI $<12$ months).

In fact, patients with de novo resistance were excluded from most studies with CDK4/6 inhibitors or represented in a small percentage; only $22 \%$ of patients with DFI of 12 months or less were included in the Paloma 2 trial, $2 \%$ in the Monaleesa2, while in Monarch3 patients were classified on a treatment-free interval longer than or shorter than 36 months.

For this reason it is important that future studies can better highlight the role of this targeted treatment especially in de novo-resistant breast cancer to personalize treatments as much as possible.

Moreover, if we consider metastatic breast cancer as a chronic disease where we have to develop a treatment strategy to optimize outcomes, we have no data to decide which is the ideal treatment algorithm for luminal breast cancer and the optimal time for incorporation of CDK4/6 inhibitors in the treatment algorithm. Until now we have no data on the optimal duration of targeted treatments; should CDK4/6 inhibitors be administered beyond progression or should they be given again after progression of one?

Finally, we do not even know which treatment option is most effective for those patients who progress after CDK4/6 inhibitors.

There is a general agreement on the early use of CDK $4 / 6$ inhibitors in the treatment of metastatic breast cancer, due to the greater PFS advantage reported in first-line setting of the disease [13].

However, some patients do not need a combination approach and perform very well with endocrine therapy alone such as fulvestrant high dose - such as those with long DFI intervals on adjuvant and first-line therapy, those with de novo, bone-only disease [14]. For these patients CDK4/6 inhibitors could represent an overtreatment. 
Moreover, since the choice of treatment strategy for HR-positive metastatic breast cancer is based almost exclusively on clinical parameters, such as DFI, number and location of metastases, and symptoms, it is important that future clinical research focuses on the identification of molecular biomarkers in order to target treatments as much as possible and to save costs and lower toxicity.

\section{Acknowledgments}

The authors thank Mr Antonio Guadalupi for support and assistance with the preparation of the manuscript.

\section{Financial \& competing interests disclosure}

The authors have no relevant affiliations or financial involvement with any organization or entity with a financial interest in or financial conflict with the subject matter or materials discussed in the manuscript. This includes employment, consultancies, honoraria, stock ownership or options, expert testimony, grants or patents received or pending, or royalties.

No writing assistance was utilized in the production of this manuscript.

\section{References}

1. Clarke R, Tyson JJ, Dixon JM et al. Endocrine resistance in breast cancer - an overview and update. Mol. Cell. Endocrinol. 418, 220-234 (2015).

2. Fedele P, Orlando L, Schiavone P et al. Recent advances in the treatment of hormone receptor positive HER2negative metastatic breast cancer. Crit. Rev. Oncol. Hematol. 94, 291-301 (2015).

3. Murphy CG, Dickler MN. The role of CDK4/6 inhibition in breast cancer. Oncologist 20, 483-490 (2015).

4. Hamilton E, Infante JR. Targeting CDK4/6 in patients with cancer. Cancer Treat. Rev. 45, 129-138 (2016).

5. Finn RS, Martin M, Rugo HS et al. Palbociclib and letrozole in advanced breast cancer. N. Engl. J. Med. 375, 1925-1936 (2016).

6. Hortobagyi GN, Stemmer SM, Burris HA et al. Ribociclib as first-line therapy for HR-positive, advanced breast cancer. N. Engl. J. Med. 375(18), 1738-1748 (2016).

7. Goetz MP, Toi M, Campone M. MONARCH 3: abemaciclib as initial therapy for advanced breast cancer. J. Clin. Oncol. 35(32), 3638-3646 (2017).

8. Cristofanilli M, Turner NC, Bondarenko I $e$ al. Fulvestrant plus palbociclib versus fulvestrant plus placebo for treatment of hormone-receptor-positive, HER2-negativemetastatic breast cancer that progressed on previous endocrine therapy (PALOMA-3): final analysis of the multicentre, double-blind, Phase III randomised controlled trial. Lancet Oncol. 17, 425-439 (2016).

9. Sledge GW Jr, Toi M, Neven P et al. MONARCH 2: abemaciclib in combination with fulvestrant in women with HR+/HER2advanced breast cancer who had progressed while receiving endocrine therapy. J. Clin. Oncol. 35, 2875-2884 (2017).

10. Slamon DJ, Neven P, Chia SKL et al. Ribociclib (RIB) + fulvestrant (FUL) in postmenopausal women with hormone receptor-positive (HR+), HER2-negative (HER2-) advanced breast cancer (ABC): results from MONALEESA-3. J. Clin. Oncol. 36, 2465-2472 (2018).

11. Chen P, Lee NV, Hu Wet al. Spectrum and degree of CDK drug interactions predicts clinical performance. Mol. Cancer Ther. 15 , 2273-2281 (2016).

12. Tolaney SM, Lin NU, Thornton D et al. Abemaciclib for the treatment of brain metastases (BM) secondary to hormone receptor positive (HR+), HER2 negative breast cancer. J. Clin. Oncol. 35 (15 Suppl.), 1019 (2017).

13. Abraham J, Coleman R, Elias A. Use of cyclin-dependent kinase (CDK) 4/6 inhibitors for hormone receptor-positive, human epidermal growth factor receptor 2-negative, metastatic breast cancer: a roundtable discussion by The Breast Cancer Therapy Expert Group (BCTEG). Breast Cancer Res. Treat. 171(1), 11-20 (2018).

14. Robertson JF, Bondarenko IM, Trishkina E et al. Fulvestrant $500 \mathrm{mg}$ versus anastrozole $1 \mathrm{mg}$ for hormone receptor-positive advanced breast cancer (FALCON): an international, randomised, double-blind, Phase III trial. Lancet 388, 2997-3005 (2016). 
